\title{
A novel approach to improve hamstring flexibility: A single-blinded randomised clinical trial
}

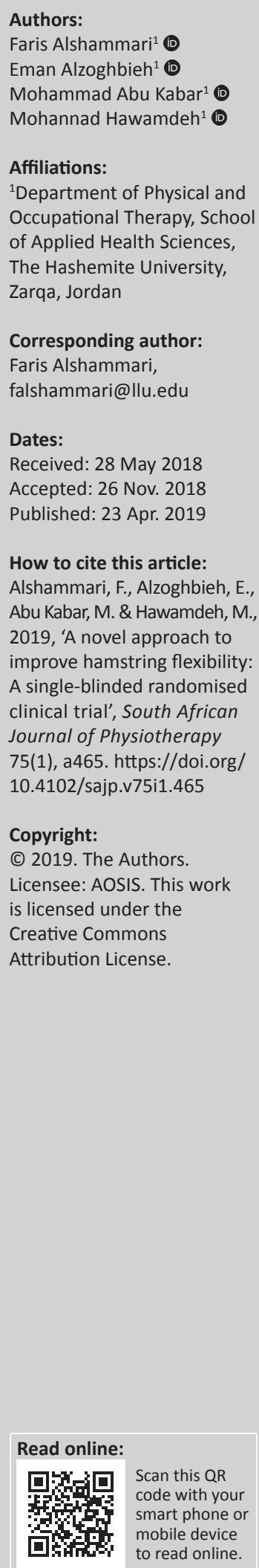

Background: The hamstrings play a major role in body posture. Shortening or tightness of the hamstrings affects postural alignment and results in possible musculoskeletal pain.

Objectives: The aim of this study was to develop a novel approach to improve hamstring flexibility in young adults.

Method: A single-blinded randomised clinical trial included 60 participants aged 18-24 with shortened hamstrings recruited from the Hashemite University, Zarqa, Jordan. The range of motion of knee extension was measured with the hip at $90^{\circ}$ flexion using a simple goniometer to detect the level of hamstring flexibility. Participants received either a passive hamstring stretch (PS), followed by two sets of 10 tibial nerve neurodynamic technique (ND), or PS followed by three sets of 10 repetitions of active knee extension-quadriceps activation (QA), or PS only.

Results: There was a significant improvement of hamstring flexibility in the QA group compared to the PS group $\left(13.4 \pm 12.1^{\circ}\right.$ vs. $\left.6.2 \pm 6.4^{\circ}, p=0.05\right)$. There was a significant improvement in hamstring flexibility post-intervention compared to pre-intervention in the PS group by $6.2 \pm 6.4\left(30.5 \pm 10.8^{\circ}\right.$ vs. $\left.36.6 \pm 9.5^{\circ}, p=0.001\right)$, ND group by $9.3 \pm 6.2\left(26.7 \pm 10.9^{\circ}\right.$ vs . $\left.36.0 \pm 9.5^{\circ}, p=0.001\right)$ and QA group by $13.4 \pm 12.1\left(20.3 \pm 9.0^{\circ}\right.$ vs. $\left.33.4 \pm 8.9^{\circ}, p=0.001\right)$.

Conclusion: Quadriceps muscle activation following passive stretching of the hamstrings appears to be superior to the PS and ND techniques in improving hamstring muscle flexibility.

Clinical implications: Quadriceps activation following passive hamstring stretching can be used in physiotherapy settings to improve hamstring muscle flexibility.

Keywords: hamstring; flexibility; stretch; neurodynamic; quadriceps; activation.

\section{Introduction}

The hamstrings is major muscles that control the movement of the hip and knee joints (Bregenhof et al. 2018;Malfait et al. 2016;Pinniger, Steele \&Groeller 2000) and control the alignment of the pelvis and spine (Jozwiak, Pietrzak \&Tobjasz 1997). So they play an important role in postural alignment where the shortening of the hamstrings could result in a posterior pelvic tilt and hypolordosis of the lumbar spine (Borman, Trudelle-Jackson \& Smith 2011;Jozwiak et al. 1997).

The changes in body posture resulting from hamstring shortening could result in lower back and lower limb pain including hip, knee or ankle joint pain (Jozwiak et al. 1997;Radwan et al. 2014; Sadler et al. 2017; Sanchez-Zuriaga, Artacho-Perez \& Bivia-Roig 2016;Witvrouw et al. 2001). A strong relationship has been shown between limited hamstring flexibility and the incidence of low back pain (Sadler et al. 2017). Similarly there appears to be a significant relationship between limited flexibility of the hamstrings and quadriceps and patellar tendinitis and tendinopathy (Morton et al. 2017; Witvrouw et al. 2001).

The prevalence of hamstring muscle tightness is fairly high (Nishchal Ratna Shakya 2018) and appears to be increasing among the youth as is shown in hamstring tightness among undergraduate physical therapy students in Nepal at $40.17 \%$ (Nishchal Ratna Shakya 2018). Because hamstring tightness affects body posture, resulting in musculoskeletal pain, it is important to develop a new effective way to improve hamstring flexibility(Borman et al. 2011;Jozwiak et al. 1997).

Many studies have been conducted on hamstring muscle stretching techniques and flexibility (Cini, De Vasconcelos \& Lima 2017; Cipriani et al. 2012; Freitas et al. 2015; Nishikawa et al. 2015). Cini et al. (2017) studied the effects of different periods of passive stretching of the hamstrings 
on hip and knee joint flexibility by comparing one group that received 30 seconds of passive stretching and a second group that received 60 seconds of passive stretching. There was a significant improvement in knee and hip flexibility within both groups, but there was no significant difference in hip or knee flexibility between the groups (Cini et al. 2017), indicating that a 30 seconds stretch is as effective as a 60 seconds stretch. Cipriani et al. (2012) studied the effect of gender and stretch frequency on hamstring muscle flexibility, finding that stretching of the hamstrings three times per week was as effective as stretching once daily. They also did not find a difference in hamstring flexibility improvement between genders (Cipriani et al. 2012).

A longer duration of hamstring stretching is important in reducing the passive torque of the hamstrings, which results in an improvement of hamstring flexibility (Freitas et al. 2015). Johnson et al. (2014) compared stretches of 10 seconds with 9 repetitions or 30 seconds with three repetitions over a 6-week period. They found that the hamstring flexibility increased with 90 seconds of stretching regardless of the duration of the stretch or the number of repetitions (Johnson et al. 2014).

Nishikawa et al. (2015) found that passive stretching was more effective than active stretching in increasing hamstring flexibility (Nishikawa et al. 2015). Self-myofascial stretching improves hamstring flexibility, and applying ultrasound prior to stretching has no effect on hamstring muscle flexibility(Cho \& Kim 2016).

Passive stretching of hamstring muscles improves flexibility. However, the use of nerve gliding or quadriceps muscle activation in conjunction with passive stretching of hamstrings could result in more flexibility. In fact, hamstring flexibility may reduce the risk of musculoskeletal pain. Therefore, it is important to develop more effective ways to improve hamstring flexibility.

The purpose of this study was to develop a new effective approach to improve hamstring flexibility. In this study, a tibial nerve neurodynamic technique (ND) and passive hamstring stretching (PS) were compared with active knee extension-quadriceps activation (QA) and PS.

\section{Methods}

A single-blinded randomised clinical trial included participants assigned randomly into three treatment groups using a blocked design with random distribution. Between April 2017 and July 2017, a total of 60 participants between 18 and 24 years old were recruited from students at the Hashemite University. Participants were recruited using flyers. They were included in this study if they had limited flexibility in their hamstrings, defined as a limitation in knee extension of $20^{\circ}$ or more, with $90^{\circ}$ hip flexion. Participants were healthy and had no history of low back or lower limb injuries. They were excluded from the study if they had a history of lower back, hip joint or knee joint pathologies.
The first author screened the participants prior to participation in order to assure compliance with the inclusion and exclusion criteria.

\section{Measurement of hamstring muscle flexibility}

Hamstring muscle flexibility was measured through the degree of limitation in the knee extension range of motion (ROM). A double-arm universal goniometer (UG) (Baseline, Albany, NY, USA) was used to measure the knee extension ROM. The UG is a valid and highly reliable tool in measuring knee joint ROM (Brosseau et al. 2001). The inter-tester reliability of the UG is $0.977-0.982$ and the intratester reliability is $0.972-0.985$. The pre- and post-intervention measures for each participant were taken by the same physiotherapist.

Participants were placed in supine, holding their hip joint at $90^{\circ}$ flexion (Figure 1). Following that, the participants were asked to extend their knee actively to their maximum ability while keeping the hip joint blocked at $90^{\circ}$ flexion. Knee extension ROM was measured at this point to determine the level of hamstring flexibility. The hamstrings were considered to have limited flexibility if the ROM limitation was $20^{\circ}$ or more (Feland et al. 2001; Hamid, Ali \& Yusof 2013). Assessors were blinded to each participant's allocation group to avoid any bias towards a specific intervention.

\section{Intervention}

The intervention was conducted by three physiotherapists trained in neurodynamic and quadriceps activation techniques. The intervention included three different techniques as follows:

1) Passive hamstring stretch: Participants were placed in supine while the hip was maintained at $90^{\circ}$ flexion. Passive knee extension done by the therapist was applied until the patient reached the maximum level of tolerable stretch. The stretch was sustained for 30 seconds each time. Each patient received three repetitions of passive stretch (Bandy \& Irion 1994; Kisner 2012).

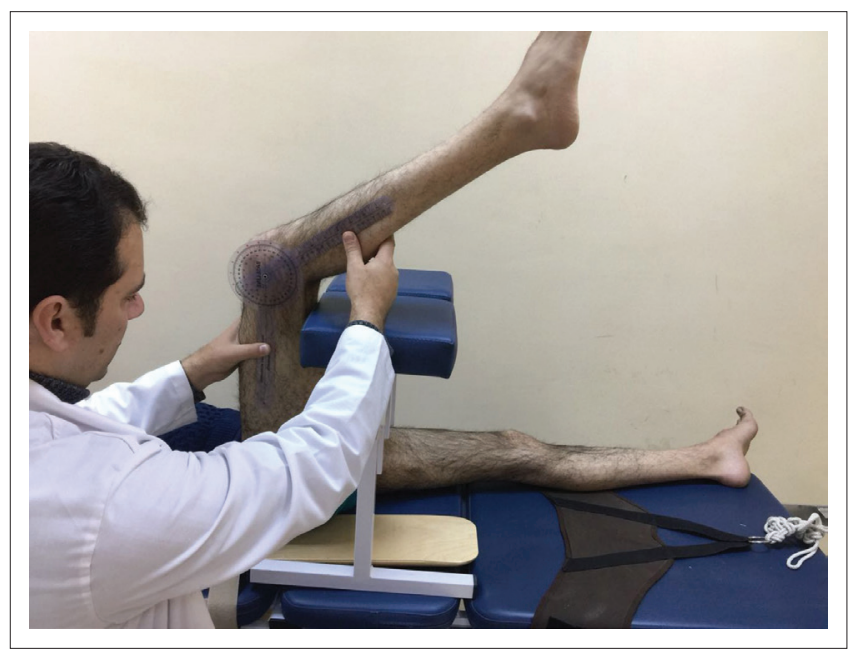

Source: Photo courtesy of the authors

FIGURE 1: Measuring hamstring muscle flexibility through knee extension range of motion. 
2) Tibial nerve neurodynamic technique following PS: Participants received PS. Following that, participants were placed in supine with the hip flexed and the knee extended. Then repetitive ankle dorsiflexion was conducted with eversion in synchronisation with knee flexion-extension in order to apply a sliding-gliding mechanism on the tibial nerve. Two sets of 10 repetitions were applied to glide the tibial nerve through manipulating the ankle and knee positions (Butler 2005).

3) Quadriceps activation following PS: Participants received PS. Following that, participants were asked to extend their knee joint actively while the hip was in $90^{\circ}$ flexion. Active knee extension was applied for three sets of 10 repetitions (Kisner 2012).

Following these procedures, hamstring muscle flexibility was measured as was done prior to the intervention.

\section{Statistical analysis}

Data were analysed using the Statistical Package for the Social Sciences version 21. The general characteristics of the participants were summarised using means and standard deviations (SDs) for quantitative variables and frequencies and percentages for categorical variables. A mixed factorial analysis of variance and one-way repeated-measures analysis of variance were used to examine the difference in mean hamstring muscle flexibility (knee ROM) between and within participant groups. Cohen's $d$ was used to determine effect sizes. The level of significance was set at 0.05 .

\section{Ethical considerations}

All protocols and procedures were approved by the Institutional Review Board of the Hashemite University (approval number RA/222/1703674). All participants signed a statement of informed consent after the study procedures were explained in detail by the first author.

\section{Results}

The demographics of the participants are shown in Table 1. There were no significant differences in age, height or weight among groups. There was no significant difference in baseline measurements among groups.

There was a significant improvement in hamstring flexibility in the group that received QA following PS compared to the group who received PS only $\left(13.4 \pm 12.1^{\circ}\right.$ vs. $6.2 \pm 6.4^{\circ}, p<$ $0.05,95 \%$ confidence interval (CI): 0.1-13.4 ${ }^{\circ}$; Figure 2). There was a significant improvement in hamstring flexibility postintervention in PS compared to pre-intervention where the knee extension increased on average by $6.2 \pm 6.4^{\circ}(30.5 \pm 10.8$ vs. $36.6 \pm 9.5^{\circ}, 95 \%$ CI: $29.6-37.5^{\circ}, p<0.001$; Figure 3). There was a significant improvement in hamstring flexibility postintervention in the ND group compared to pre-intervention where the knee extension increased by $9.3 \pm 6.2^{\circ}\left(26.7 \pm 10.9^{\circ}\right.$ vs. $36.0 \pm 9.5^{\circ}, 95 \% \mathrm{CI}: 27.4-35.3^{\circ}, p<0.001$; Figure 4). There was a significant improvement in hamstring flexibility
TABLE 1: Demographic characteristics by study group $(n=60)$.

\begin{tabular}{lcccc}
\hline Variable & Neurodynamic & Quad activation & Passive stretching & $\boldsymbol{p}$ \\
\hline Age (years) & $21.5 \pm 0.9$ & $21.7 \pm 0.8$ & $21.5 \pm 0.6$ & 0.657 \\
Weight $(\mathrm{kg})$ & $63.7 \pm 10.2$ & $62.1 \pm 10.9$ & $64.5 \pm 10.8$ & 0.752 \\
Height $(\mathrm{cm})$ & $166.7 \pm 11.9$ & $168.4 \pm 12.1$ & $169.8 \pm 11.9$ & 0.749 \\
\hline
\end{tabular}

SD, standard deviation.

Data are mean \pm SD values.

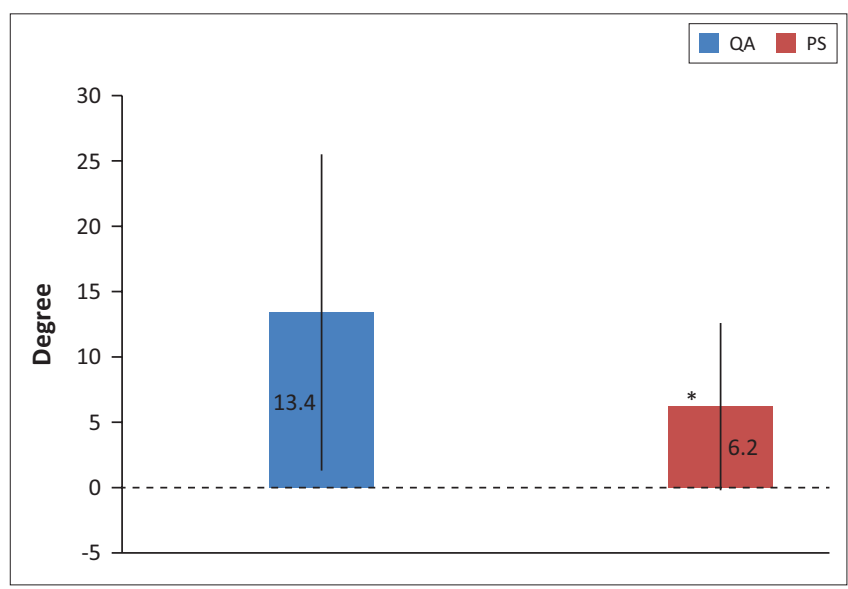

$*, p<0.05$.

$\mathrm{QA}$, quadriceps activation; PS, passive hamstring stretch.

FIGURE 2: Mean improvement of hamstring muscle flexibility (in degrees) \pm standard deviation between quadriceps activation and passive stretch groups.

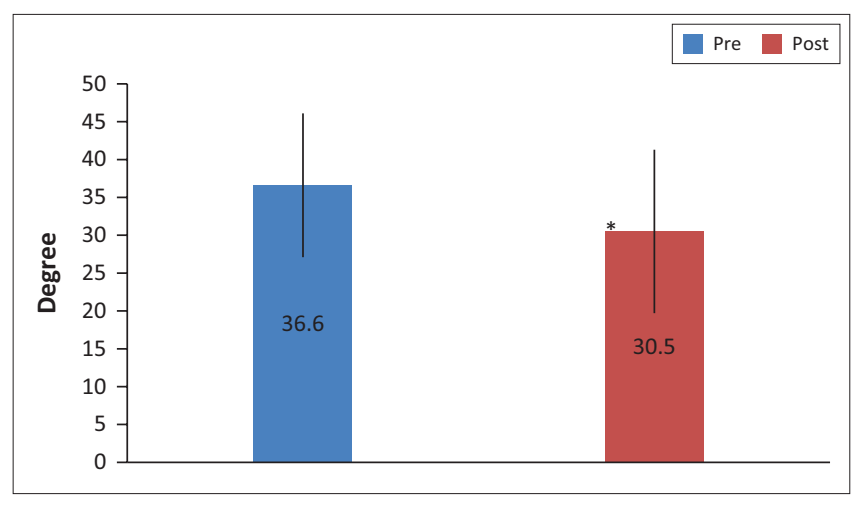

$*, p<0.001$.

FIGURE 3: Mean knee extension range of motion (in degrees) \pm standard deviation pre- and post-intervention in the group that received passive stretching.

post-intervention in the QA group compared to preintervention where the knee extension increased by $13.4 \pm$ $12.1^{\circ}\left(20.3 \pm 9.0^{\circ}\right.$ vs. $33.4 \pm 8.9^{\circ}, 95 \%$ CI: $22.8-30.7^{\circ}, p<0.001$; Figure 5). Cohen's effect indicated the presence of a moderate effect size for ND compared to PS (Cohen's $d=0.5)$.

\section{Discussion}

The group that received quadriceps activation following passive hamstring stretching improved significantly compared to the group that received passive stretching only. This could be because of the reciprocal inhibition effect (Crone 1993). Reciprocal inhibition occurs through a spinal cord primitive reflex arc when the antagonist muscle relaxes in response to the activation of the agonist muscle. Therefore, the group that had the quadriceps activation had more inhibition to the antagonist muscle (hamstring) compared to the group that only received passive stretching. 


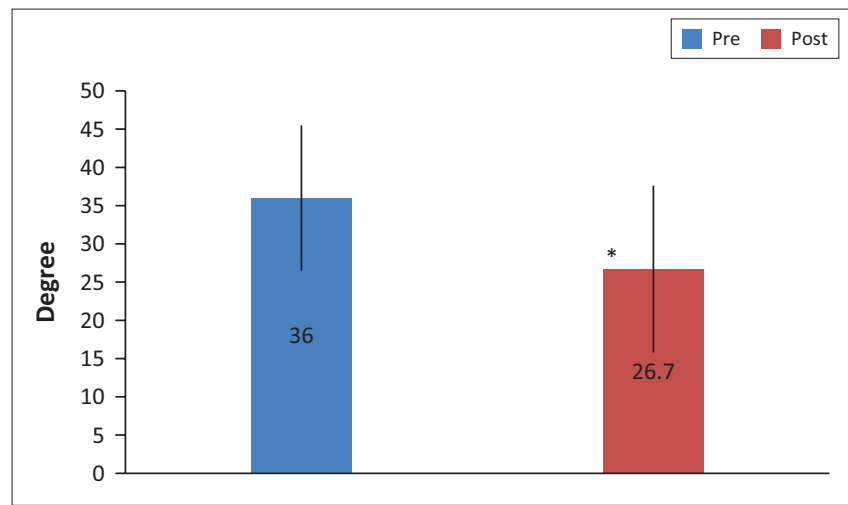

$*, p<0.001$.

FIGURE 4: Mean knee extension range of motion (in degrees) \pm standard deviation pre- and post-intervention in the group that received neurodynamic technique following passive stretching.

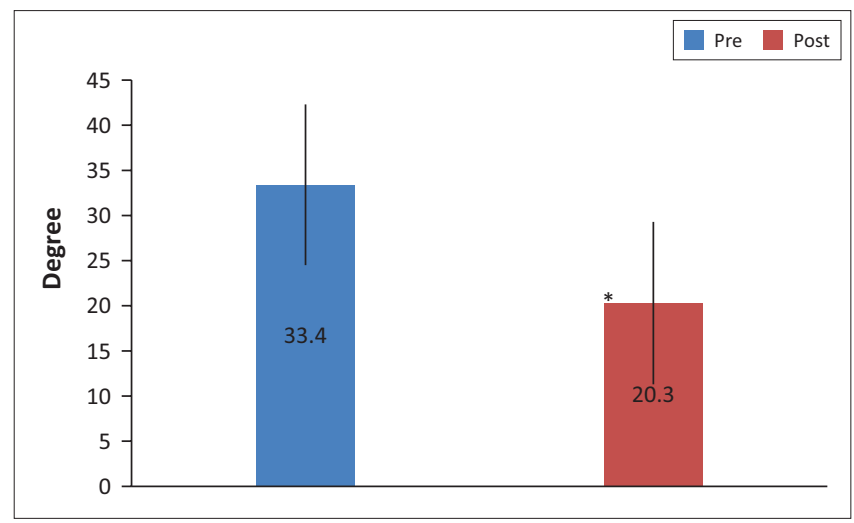

$*, p<0.001$.

FIGURE 5: Mean knee extension range of motion (in degrees) \pm standard deviation pre- and post-intervention in the group that received quadriceps activation following passive stretching.

Nishikawa et al. (2015) studied the immediate effect of passive stretching compared to active stretching on hamstring muscle flexibility. They found that passive stretching was more effective than active stretching in increasing hamstring muscle flexibility (Nishikawa et al. 2015). This is contrary to the findings of our study; however, they did not precede active stretching with passive stretching as was done in our study. Passive stretching prior to active stretching could prepare the muscle for reciprocal inhibition and result in better outcomes.

There was no significant difference in hamstring muscle flexibility between the ND group and PS group. This could be a result of the fact that participants received two sets of 10 repetitions of the neurodynamic technique, which may have not been sufficient to create a statistical difference between groups. Therefore, a future study is recommended to include three sets of 10 repetitions of the neurodynamic technique to determine the effect on hamstring flexibility. Even though the difference between the ND group and PS group was not statistically significant, it was clinically important. Cohen's effect indicated the presence of a moderate effect size for the neurodynamic technique compared to passive stretch (Cohen's $d=0.5$ ). This supports the idea of having more repetitions of the neurodynamic technique in order to have statistically significant differences between groups.
Bandy and Irion (1994) and Cipriani et al. (2012) found that 30 seconds of passive stretch is as effective as 60 seconds of passive stretch (Bandy \& Irion 1994; Cipriani et al. 2012), supporting the findings of our study. In our study, the group who received 30 seconds passive stretch had significant improvements in hamstring flexibility post-intervention compared to pre-intervention. Similarly Johnson et al. (2014) support our findings, as they found a significant improvement in hamstring muscle flexibility following passive stretching (Johnson et al. 2014).

Our findings may be helpful in physiotherapy clinical settings where there is a need to improve hamstring flexibility. Physiotherapists can combine passive stretching with the quadriceps activation technique in order to achieve the optimal effect on hamstring flexibility in patients who have tight hamstrings. This may help in reducing the risk of having postural changes that could result in musculoskeletal pain and dysfunction (Borman et al. 2011; Jozwiak et al. 1997; Radwan et al. 2014; Sadler et al. 2017).

The limitations of this study include the small sample size, unequal repetitions of neurodynamic and quadriceps activation techniques and a lack of carry-over measurements. Future studies are recommended to find the effect and carryover of repetitive use of neurodynamic and quadriceps activation techniques on hamstring muscle flexibility with a bigger sample size and more repetitions of the neurodynamic technique.

\section{Conclusion}

Quadriceps muscle activation following passive stretching of hamstring muscle is an effective way to improve hamstring flexibility.

\section{Clinical implications}

Quadriceps activation following passive hamstring stretching can be used in physiotherapy settings to improve hamstring flexibility in people who have tight hamstrings, resulting in postural changes or musculoskeletal pain.

\section{Acknowledgements}

I would like to acknowledge the Physical and Occupational Therapy Department at the Hashemite University for the help and support that they provided.

\section{Competing interests}

The authors declare that they have no financial or personal relationships that may have inappropriately influenced them in writing this article.

\section{Authors' contributions}

F.A. was the project leader. F.A. and E.A. were responsible for study idea and study protocol. F.A., E.A., and M.H. were responsible for data analysis and data interpretation. F.A. 
and M.A.K were responsible for ethical approval and data collection. F.A., E.A., M.A.K. and M.H were responsible for writing paper.

\section{Disclaimer}

The views expressed in this article are the authors' own and not an official position of the Hashemite University or Jordanian University.

\section{References}

Bandy, W.D. \&Irion, J.M., 1994,'The effect of time on static stretch on the flexibility of the hamstring muscles', Physical Therapy 74, 845-850; discussion 850-852. https://doi.org/10.1093/ptj/74.9.845

Borman, N.P., Trudelle-Jackson, E. \&Smith, S.S., 2011،'Effect of stretch positions on hamstring muscle length, lumbar flexion range of motion, and lumbar curvature in healthy adults', Physiotherapy: Theory and Practice 27, 146-154.

Bregenhof, B., Jorgensen, U., Aagaard, P., Nissen, N., Creaby, M.W., Thorlund, J.B. et al., 2018, 'The effect of targeted exercise on knee-muscle function in patients with persistent hamstring deficiency following $\mathrm{ACL}$ reconstruction - Study
protocol for a randomized controlled trial', Trials 19, 75. https://doi.org/10.1186/ protocol for a randomiz-018-2448-3

Brosseau, L., Balmer, S., Tousignant, M., O'Sullivan, J.P., Goudreault, C., Goudreault, M. et al., 2001, 'Intra- and intertester reliability and criterion validity of h. the parallelogram and universal goniometers for measuring maximum active

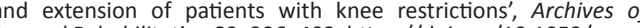
Physical Medicine and Rehabilitation 82, 396-402. https://doi.org/10.1053/apmr. 2001.19250

Butler, D., 2005, The neurodynamic techniques: A definitive guide from the Noigroup team, NOI Group Publications, Adelaide City West, South Australia, pp. 10-16.

Cho, S.H. \& Kim, S.H., 2016, 'Immediate effect of stretching and ultrasound on hamstring flexibility and proprioception', Journal of Physical Therapy Science 28, 1806-1808. https://doi.org/10.1589/jpts.28.1806

Cini, A., DeVasconcelos, G.S. \& Lima, C.S., 2017, 'Acute effect of different time periods of passive static stretching on the hamstring flexibility', Journal of Back and Musculoskeletal Rehabilitation 30, 241-246. https://doi.org/10.3233/BMR160740

Cipriani, D.J., Terry, M.E., Haines, M.A., Tabibnia, A.P. \& Lyssanova, O., 2012, 'Effect of stretch frequency and sex on the rate of gain and rate of loss in muscle flexibility during a hamstring-stretching program: A randomized single-blind longitudinal study', Journal of Strength and Conditioning Research 26, 2119-2129. https://doi. study', Journal of Strength and Condition

Crone, C., 1993, 'Reciprocal inhibition in man', Danish Medical Bulletin 40, 571-581.

Feland, J.B., Myrer, J.W., Schulthies, S.S., Fellingham, G.W. \& Measom, G.W., 2001,'The effect of duration of stretching of the hamstring muscle group for increasing range of motion in people aged 65 years or older', Physical Therapy 81, 1110-1117.
Freitas, S.R., Vilarinho, D., Rocha Vaz, J., Bruno, P.M., Costa, P.B. \& Mil-Homens, P. 2015, 'Responses to static stretching are dependent on stretch intensity and 2015, 'Responses to static stretching are dependent on stretch intensity and
duration', Clinical Physiology and Functional Imaging 35, 478-484. https://doi. org/10.1111/cpf.12186

Hamid, M.S., Ali, M.R. \& Yusof, A., 2013, 'Interrater and intrarater reliability of the active knee extension (AKE) test among healthy adults', Journal of Physical Therapy Science 25, 957-961. https://doi.org/10.1589/jpts.25.957

Johnson, A.W., Mitchell, U.H., Meek, K. \& Feland, J.B., 2014,'Hamstring flexibility increases the same with 3 or 9 repetitions of stretching held for a total time of 90s', Physical Therapy in Sport 15, 101-105. https://doi.org/10.1016/j.ptsp. 2013.03.006

Jozwiak, M., Pietrzak, S. \& Tobjasz, F., 1997, 'The epidemiology and clinical manifestations of hamstring muscle and plantar foot flexor shortening', Developmental Medicine \& Child Neurology 39, 481-483. https://doi.org/10. 1111/j.1469-8749.1997.tb07468.x

Kisner, C., 2012, Therapeutic exercise: Foundations and techniques, F. A. Davis Co., Philadelphia, PA.

Malfait, B., Dingenen, B., Smeets, A., Staes, F., Pataky, T., Robinson, M.A. et al., 2016 'Knee and hip joint kinematics predict quadriceps and hamstrings neuromuscular activation patterns in drop jump landings', PLoS One 11, e0153737. https://doi. org/10.1371/journal.pone.0153737

Morton, S., Williams, S., Valle, X., Diaz-Cueli, D., Malliaras, P. \& Morrissey, D., 2017 'Patellar tendinopathy and potential risk factors: An international database of
cases and controls', Clinical Journal of Sport Medicine 27, 468-474. https://doi. org/10.1097/JSM.0000000000000397

Nishchal Ratna Shakya, S.M., 2018, 'Prevalence of hamstring muscle tightness among undergraduate physiotherapy students of Nepal using passive knee extension angle test', International Journal of Scientific and Research Publications 8, angle test',

Nishikawa, Y., Aizawa, J., Kanemura, N., Takahashi, T., Hosomi, N., Maruyama, H. et al. 2015, 'Immediate effect of passive and active stretching on hamstrings flexibility: A single-blinded randomized control trial', Journal of Physical Therapy Science 27 , 3167-3170. https://doi.org/10.1589/jpts.27.3167

Pinniger, G.J., Steele, J.R. \& Groeller, H., 2000, 'Does fatigue induced by repeated dynamic efforts affect hamstring muscle function?', Medicine \& Science in Sports \& Exercise 32, 647-653. https://doi.org/10.1097/00005768-200003000-00015

Radwan, A., Bigney, K.A., Buonomo, H.N., Jarmak, M.W., Moats, S.M., Ross, J.K. et al., 2014,'Evaluation of intra-subject difference in hamstring flexibility in patients with low back pain: An exploratory study', Journal of Back and Musculoskeletal Rehabilitation 28(1), 61-66.

Sadler, S.G., Spink, M.J., Ho, A., De Jonge, X.J. \& Chuter, V.H., 2017,'Restriction in lateral bending range of motion, lumbar lordosis, and hamstring flexibility predicts the development of low back pain: A systematic review of prospective cohort studies', BMC Musculoskeletal Disorders 18, 179. https://doi.org/10.1186/ s12891-017-1534-0

Sanchez-Zuriaga, D., Artacho-Perez, C. \& Bivia-Roig, G., 2016, 'Lumbopelvic flexibility modulates neuromuscular responses during trunk flexion-extension', Journal of Electromyography and Kinesiology 28, 152-157. https://doi.org/10.1016/j. jelekin.2016.04.007

Witvrouw, E., Bellemans, J., Lysens, R., Danneels, L. \& Cambier, D., 2001, 'Intrinsic risk factors for the development of patellar tendinitis in an athletic population. A twoyear prospective study', American Journal of Sports Medicine 29, 190-195. https://doi.org/10.1177/03635465010290021201 